\title{
A Survey Paper of a Distributed Three-hop Routing Protocol to Increase the Capacity of Hybrid Wireless Networks
}

\author{
Pallavi T. Dhotre ${ }^{1}$, Mohasin B. Tamboli ${ }^{2}$ \\ M.E. Student, G. H. Raisoni College of Engineering and Management, Ahmednagar,414001,India \\ Associate Professor, G. H. Raisoni College of Engineering and Management, Wagholi, Pune,411015,India
}

\begin{abstract}
Hybrid Wireless networks combining the advantages of mobile ad-hoc networks and wireless networks has been increased attention due to their high performance. In now days, wireless sensor networks applications are used in various technologies for reducing the cost of manufacturing portable wireless sensor nodes. Hybrid WSN needs to provide an assured Quality of Service (QOS) in application. To increase QOS large number of portable sensor nodes are developed. This paper represents Distributed Three-hop Routing protocol (DTR) for hybrid wireless networks. This takes advantage of the wireless networks where DTR divides message data stream into each segments and transmits these segments in a distributed manner.
\end{abstract}

Keywords: Hybrid wireless network, Distributed Three-hop Routing protocol, wireless networks, MANET, QOS

\section{Introduction}

In the hybrid WSN, node of energy consumption is important for every sensor node because it extends hybrid WSN life. The Wireless sensor network is a collection of all sensors which spread over huge geographic area. As sensors are spread in large area and huge in number, the occurrences of faults in the network are also find. Hence to find out the fault node and to replace the fault node an algorithm is proposed.

This paper proposes different algorithm to increase the lifetime of a hybrid wireless sensor networks when some of the sensor nodes fail down using the algorithm can result in some replacements of sensor nodes and used routing path. Thus, the algorithm enhances the hybrid WSN lifetime and reduces the change of the sensor nodes.

A hybrid wireless network combination of a mobile ad-hoc network and an infrastructure wireless network and finally enhances the capacity of a wide area wireless network. Routing protocol is an important component that affects the strength of a wireless network in data transmission. Routing path in hybrid wireless networks combination of the cellular Transmission Mode (BSTransmission Mode) in Ad-Hoc transmission mode and infrastructure wireless networks the in mobile ad- hoc networks. The below subsection will give information about Algorithm used in Distributed Three-hop Routing Protocol are:

\section{Load Balancing Algorithm:}

It propose a load balancing scheme called iCAR for cellular networks, which places ad hoc relay nodes at strategic locations to relay traffic from congested cells to noncongested ones.

\section{Wireless Network with RRP algorithm:}

It consider the Multistage Multiplane Clos-Network based switch by Chao et a. It is designed IN five stages of switch modules with top-level architecture same as to external input or output ports. The first and final stages Clos are contain of input De-Multiplexers and output multiplexers, having similar internal structures and various wireless sensors. This algorithm generates the grade number and routing table, a set of acquaintance nodes and payload value each sensor node.

3. DTR: Distributed Three-hop Routing (i.e. DTR) Data Routing Protocol that improves the features of hybrid wireless networks in the data transmission process. In DTR, a source node is divides a message stream into segments and transmits it into its mobile neighbors, which again forward the segments to destination through an infrastructure network path.

Advantages of different algorithm are as follows:

- The main aim for Quality of Service (QOS) is to reduce failure of a sensor node.

- In order to increase the capacity of hybrid wireless networks, various routing methods with different features are implemented.

- Wireless sensor can be replaced in case of failure by using algorithm.

The below table shows comparison between each algorithm

\begin{tabular}{|c|c|c|c|}
\hline $\begin{array}{c}\text { Algorithm/ } \\
\text { Parameters }\end{array}$ & $\begin{array}{c}\text { Load } \\
\text { Balancing } \\
\text { Algorithm }\end{array}$ & $\begin{array}{c}\text { Wireless } \\
\text { Network with } \\
\text { RRP algorithm }\end{array}$ & DTR \\
\hline Working & $\begin{array}{c}\text { places ad } \\
\text { hoc relay } \\
\text { nodes at } \\
\text { strategic } \\
\text { locations }\end{array}$ & $\begin{array}{c}\text { algorithm } \\
\text { generates the } \\
\text { grade number } \\
\text { and routing } \\
\text { table }\end{array}$ & $\begin{array}{c}\text { source node is } \\
\text { divides a } \\
\text { message } \\
\text { stream into } \\
\text { segments }\end{array}$ \\
\hline Efficiency & Low & Average & High \\
\hline $\begin{array}{c}\text { Time } \\
\text { Complexity }\end{array}$ & High & High & High \\
\hline performance & Low & medium & High \\
\hline
\end{tabular}




\section{International Journal of Science and Research (IJSR) \\ ISSN (Online): 2319-7064}

Index Copernicus Value (2013): 6.14 | Impact Factor (2014): 5.611

\section{Related Work}

1) Ucan: A Unified Cell and Ad-hoc Network architecture [2].This paper presents a Unified Cellular and Ad-hoc Network (UCAN) structure to increase cell throughput. In UCAN, a mobile client hasIEEE 802.11 based peer topeer linksand3G cellular link. The $3 \mathrm{G}$ cellularbase stations distribute packets to destination station with low channel quality to proxy clients. The proxy clients use an Ad-Hoc network made up of other mobile clients and IEEE 802.11 wireless links to distribute the packets to the destinations station. This paper further represents secure relayed packets for other stations. Extensive simulation with IEEE 802.11(b). We show that the UCAN architecture can gives separate user's output by up to $80 \%$ and the aggregate throughput of downlink by up to $60 \%$

2) Multi-hop cellular: This is new architecture for wireless communications [3], this paper represents a new architecture, Multihop Cellular Network (MCN) for wireless communication. MCN reserves the advantage of conventional single hop cellular network (SCN), where the service infrastructure is designed by fix bases and it also the flexible of ad-hoc networks, where wireless transmission through mobile stations in Multiple Hops are allowed. The MCN can be reducing the required number of bases to enhance the throughput performance while limiting path encountered in ad-hoc networks. In addition SCN and MCN are analyzed; in term of mean hop count, hop by hop throughput and end throughput, and mean number of channels under different.

3) Connectivity in ad hoc and hybrid networks [4].This paper shows the introduction of a sparse network of base station do significantly help in increase the connectivity, but it only when the node density is much more in one dimension than in the other. They explain the results by percolation theory. This paper obtains analysis of expressions of connectivity in the 1dimension case. They also show that at a less spatial density of nodes and bottleneck are unavoidable; results obtained on real population data confirm our finding.

4) Highly Dynamic Destination Sequenced Distance Vector routing (DSDV) for mobile computers. [5] In this paper they represented a new structure for the operation of such ad-hoc network. The basic invention of the structure is to operate every mobile host as a special router, which eventually advertising its views of the interconnection topology with another mobile hosts within the networks. That amounts to sort of routing protocol. They have investigation modified to the Bellman Ford routing mechanisms, as specific to make it compatible for dynamic and self-starting network mechanism as is required by users to utilize ad-hoc networks. It modifications address some of the objections to the use of Bellman-Ford, related to the less looping properties of algorithms in the face of broke links and the results time depends upon nature of the interconnection topology describing the link between the mobile hosts. They describe the ways in which the network layer routing can be modified to provide MAC layer support for Ad-hoc networks.
5) Ad-hoc On Demand Distance Vector (AODV) routing[6], In this paper they represent AODV algorithm for the operation of Ad-hoc networks to every mobile host operates as a some special router, and routes are obtained as need with small or no reliance on advertisements their new routing algorithm is more suitable for a dynamic self-starting network, as required by users wishing to utilize ad-hoc networks AODV provides loop routes even while repairs broken links Because the protocol do not require global routing advertisements, the demand on the bandwidth available to the mobile nodes is less than in those protocols that do necessary such advertisements. We can maintain the advantages of basic distance vector routing mechanisms in network. They show that their algorithm scales to larger populations of mobile nodes to form Ad-hoc networks it also include an evaluation methodology and simulation results to the operation of algorithm Keywords.

\section{Conclusions and Future Work}

In this paper we studied A Distributed Three-hop Routing (DTR) to increase the strength of Hybrid Wireless Networks (HWN). In this paper proposes different algorithm recovery and replacement that increases the strength when sensors nodes are fail. DTR data routing protocol that contains the features of hybrid wireless networks in the data transmission process. In DTR, a source node station divides a message stream into segments and then transmits them to its mobile neighbors, which further distributed the segment to their proper destination through an infrastructure network. This paper shows different approach of wireless senor recovery in related work. We will propose a recovery and replacement algorithm which is a combination of genetic algorithm and grade diffusion algorithm

\section{Acknowledgment}

We would like to thank all the authors of different research papers referred during writing this paper. It was very knowledge gaining and helpful for the further research to be done in future.

\section{References}

[1] A Distributed Three-hop Routing Protocol to Increase the Capacity of Hybrid Wireless Networks HaiyingShen*, Senior Member, IEEE, $\mathrm{Ze} \mathrm{Li}$ and ChenxiQiu. IEEE Transactions on Mobile Computing VOL. XX, NO.XX 2015

[2] H Luo, R. Ramjee, P. Sinha, L. Li, and S. Lu.Ucan: A unified cell and ad-hoc network architecture. In Proc. of MOBICOM, 2003.

[3] Y. D. Lin and Y. C. Hsu. Multi-hop cellular: A new architecture for wireless communications. In Proc. of INFOCOM, 2000.

[4] P. T. Oliver, Dousse, and M. Hasler. Connectivity in ad hoc and hybrid networks.In Proc. of INFOCOM, 2002.

[5] E. P. Charles and P. Bhagwat. Highly dynamic destination sequenced distance vector routing (DSDV) for mobile computers.In Proc. of SIGCOMM, 1994. 


\section{International Journal of Science and Research (IJSR) \\ ISSN (Online): 2319-7064}

Index Copernicus Value (2013): 6.14 | Impact Factor (2014): 5.611

[6] C. Perkins, E. Belding-Royer, and S. Das. RFC 3561: Ad hoc on demand distance vector (AODV) routing. Technical report, Internet Engineering Task Force, 2003.

[7] D. B. Johnson and D. A. Maltz. Dynamic source routing in ad hoc wireless networks.IEEE Mobile Computing, 1996.

[8] V. D. Park and M. Scott Corson.A highly adaptive distributed routing algorithm for mobile wireless networks. In Proc.Of INFOCOM, 1997.

[9] R. S. Chang, W. Y. Chen, and Y. F. Wen.Hybrid wireless network protocols.IEEE Transaction on Vehicular Technology, 2003.

[10]G. N. Aggelou and R. Tafazolli.On the relaying capacity of nextgeneration gsm cellular networks. IEEE Personal Communications Magazine, 2001

[11]T. Rouse, I. Band, and S. McLaughlin. Capacity and power investigation of opportunity driven multiple access (ODMA) networks in TDD-CDMA based systems. In Proc. of ICC, 2002.

[12]P. Gupta and P. R. Kumar. The capacity of wireless networks. IEEETIT, 2000

[13] J. Cho and Z. J. Haas.On the throughput enhancement of the downstream channel in cellular radio networks through multihop relaying. IEEE JSAC, 2004.

[14]B. Liu, P. Thiran, and D. Towsley.Capacity of a wireless ad hoc network with infrastructure. In Proc. of Mobihoc, 2007

[15] AzzedineBoukerche., 2009. ALGORITHMS AND PROTOCOLS FOR WIRELESS AND MOBILE AD HOC NETWORKS. Willey Publication 